\title{
Multichannel PCR-CE Microdevice for Rapid Genetic Analysis
}

\author{
Chung N. Liu ${ }^{a}$, Nicholas M. Toriello ${ }^{b}$ \& Richard A. Mathies ${ }^{b, c^{*}}$ \\ ${ }^{a}$ Department of Chemical Engineering, \\ ${ }^{\mathrm{b}}$ UCSF/UC Berkeley Joint Graduate Group in Bioengineering, \\ ${ }^{\mathrm{c}}$ Department of Chemistry,
}

University of California, Berkeley, CA 94720, USA

\section{Supporting information}

RTD calibration for the multichannel PCR-CE microdevice. Calibration curves for the four Ti/Pt RTDs on a wafer. Temperature as a function of resistance was linear-fitted to each curve with $R^{2}>0.9995$. Ti/Pt RTDs are fabricated using standard photolithographic techniques. Calibration and temperature detection is conducted with constant $4 \mathrm{~mA}$ current source. Calibration curves:

RTD 1: $T(R)=0.478 \cdot R-470$

RTD 2: $T(R)=0.481 \cdot R-402$

RTD 3: $T(R)=0.496 \cdot R-490$

RTD 4: $T(R)=0.475 \cdot R-438$

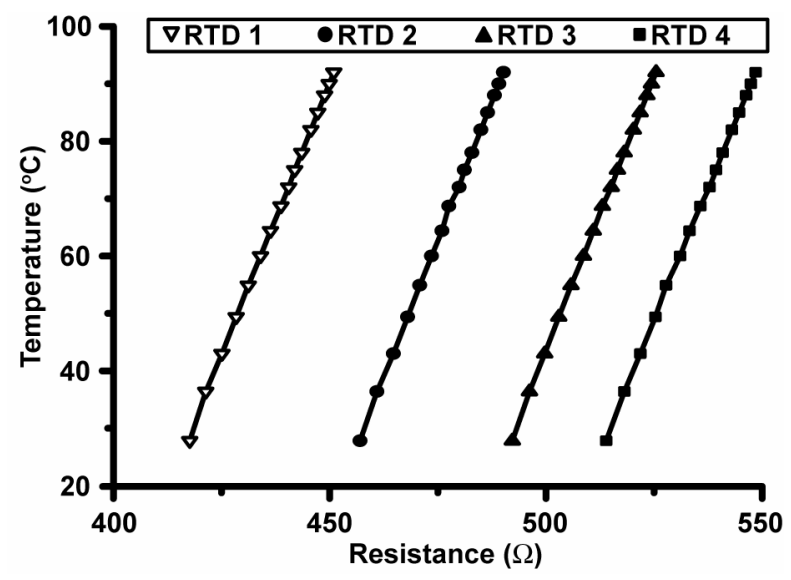

